\title{
Sobrevida global y por estadíos de 137 pacientes con cáncer intraoral. Experiencia del Instituto Nacional del Cáncer*
}

\author{
Drs. PABLO BÓRQUEZ M. ${ }^{1}$, FELIPE CAPDEVILLE F. ${ }^{1}$, ARTURO MADRID M. ${ }^{1}$, \\ MARCELO VELOSO O. ${ }^{1}$, MARCELA CÁRCAMO P. ${ }^{1}$ \\ 1 Instituto Nacional del Cáncer. \\ Santiago, Chile.
}

\begin{abstract}
Analysis of survival of 137 patients with oral cancer

Background: Oral cancer accounts for $1 \%$ of cancer mortality. It is more common in men but its frequency is increasing in women due to their growing smoking habits. Aim: To report the experience in the management of a cohort of patients with oral cancer. Material and Methods: Review of medical records of patients with oral cancer treated between 1989 and 2004. Demographic and clinical data were recorded and survival was determined examining death certificates or information obtained from the medical records. Results: Data from 137 patients aged $61 \pm 14$ years (98 males) was analyzed. Global survival five years survival was $57 \%$. Survival for stages I, II, III and IV was $86,67,52$ and 51\% respectively. Conclusions: The survival of these patients is very similar to that reported abroad.
\end{abstract}

Key words: Oral cancer, survival, smoking.

\section{Resumen}

El cáncer intraoral representa un $1 \%$ de la mortalidad por cáncer. Ocurre predominantemente en hombres aunque la frecuencia en mujeres se ha incrementado en los últimos 20 años debido al creciente consumo de tabaco en este grupo. Son cuadros complejos que requieren un manejo integrado de múltiples especialistas. El objetivo de este trabajo es presentar la experiencia del Instituto Nacional del Cáncer (INC) en el manejo de una cohorte de 137 casos tratados y seguidos en esta institución entre 1989 y 2004. La población tratada corresponde predominantemente a hombres $(71,5 \%)$ con edad promedio de 61 años. La sobrevida global fue de $57 \%$ a 5 años, por estadíos la sobrevida fue de $86 \%$ en etapa I, $67 \%$ en etapa II, $52 \%$ en etapa III y $51 \%$ en etapa IV. Estos resultados son comparables con otros centros oncológicos del extranjero y son reflejo del adecuado manejo de los pacientes.

Palabras clave: Cáncer intraoral, tratamiento, sobrevida.

\footnotetext{
* Recibido el 19 de marzo de 2010 y aceptado para publicación el 13 de diciembre de 2010.
}

Correspondencia: Dr. Pablo Bórquez M. Av. Profesor Zañartu Nº1010, fax: 56-02-5757157, Santiago, Chile. cir.oncologo@yahoo.es 


\section{Introducción}

La tasa de mortalidad del cáncer intraoral representó menos del $1 \%$ de la mortalidad general por cáncer en Chile entre 1955 y 2002, afectando al $74 \%$ de los hombres y $26 \%$ de las mujeres, con una razón de 2,8:1. En hombres, ha permanecido estable las 2 últimas décadas, sin embargo, en mujeres la tasa de mortalidad se encuentra en ascenso debido al aumento del consumo de tabaco ${ }^{1}$. En EE.UU., el cáncer intraoral es causa de un $14 \%$ de los cánceres de cabeza y cuello, con una tasa de mortalidad de un $25 \%{ }^{2}$.

Estos tumores se refieren a las lesiones malignas que se originan en los distintos subsitios de la cavidad oral como lengua, reborde alveolar, paladar, piso de boca, mucosa yugal, trígono retromolar entre otras localizaciones. El tipo histológico más frecuente es el carcinoma epidermoide en un $90 \%$ de los casos, aunque se han descrito otros tipos como el cáncer de glándulas salivales o melanoma ${ }^{3}$. Debido a la ubicación de estos tumores y a una conducta biológica variable según el subsitio afectado, son tumores de manejo altamente especializado que requieren de un abordaje multidisciplinario que incluye como tratamiento la cirugía, radioterapia y quimioterapia. No obstante, las mejoras en las técnicas de reconstrucción y rehabilitación, no han mejorado sustancialmente los resultados del tratamiento oncológico en los últimos 25 años ${ }^{1,4}$.

El objetivo de este trabajo es presentar la sobrevida global y por estadíos de 137 pacientes con cáncer intraoral tratados y seguidos en el Instituto Nacional del Cáncer (INC).

\section{Material y Método}

Se presenta una serie de casos con seguimiento compuesta por 137 pacientes consecutivos, tratados en el Instituto Nacional del Cáncer de Santiago (INC), Chile. Se incluyeron los pacientes tratados entre 1989 y 2004, de ambos sexos, sin límite de edad, con diagnóstico de cáncer intraoral confirmado por histopatología. Para la estadificación de los pacientes se empleó la clasificación TNM del American Joint Committe on Cancer (AJCC) ${ }^{5}$, TAC de cabeza y cuello para el estudio preoperatorio del tumor primario y linfonodos cervicales, además de una radiografía de tórax, evaluación nutricional y broncopulmonar preoperatoria.

En una base de datos especialmente diseñada, se recolectó la información derivada de las fichas clínicas que incluyó variables demográficas, aspectos clínicos y terapéuticos.
Se realizó estadística descriptiva de las variables tales como sexo, edad, antecedentes mórbidos, hábitos, histología, clasificación TNM, etapa, estado de los linfonodos y metástasis. Dichas variables se presentaron mediante cifras absolutas y relativas. La sobrevida se expresa mediante sobrevida actuarial con curvas de Kaplan Meyer. Para su cálculo se consideró como fecha de inicio al seguimiento, la fecha de ingreso al comité oncológico de la institución y como fecha de término la muerte obtenida mediante certificado de defunción o el estado actual del paciente obtenido desde el programa de seguimiento elaborado por el instituto INC 2.0. Para realizar la estadística descriptiva y las curvas de sobrevida se utilizó el software estadístico STATA 10.0 .

\section{Resultados}

De los 137 pacientes, 98 son hombres $(71,53 \%)$ y 39 mujeres $(28,47 \%)$. La edad promedio fue de $60,6 \pm 14,1$ años y una mediana de 62 años (2095). En relación a los antecedentes mórbidos, 112 pacientes no los presentaron $(81,2 \%), 7$ pacientes tenían hipertensión arterial $(5,1 \%), 3$ pacientes presentan diabetes mellitus $(2,2 \%), 2$ pacientes tenían silicosis $(1,5 \%)$, otras enfermedades 13 $(10 \%)$. Respecto de los hábitos, 44 pacientes son no fumadores $(32,12 \%)$ y 93 son fumadores $(67,88 \%)$; 81 pacientes son no bebedores $(59,12 \%)$ y 56 son bebedores (40,88\%).

Las ubicaciones del tumor primario y los principales tipos histológicos pueden apreciarse en la Tabla 1. La distribución de los pacientes según TNM (en que el "T" corresponde al tamaño tumoral, el "N" al estado de linfonodos cervicales y "M" a la presencia de metástasis a distancia) junto con la distribución según estadios se observa en la Tabla 2. La Tabla 3 muestra los distintos tratamientos realizados. Finalmente, la Tabla 4 ofrece un resumen de los resultados de sobrevida de distintas series clínicas extranjeras.

La sobrevida global puede apreciarse en la Figura 1 . A 5 años fue $56,9 \%$ y a 10 años fue $46,3 \%$. La sobrevida por estadíos (Figura 2) fue a 5 y 10 años de $85,7 \%$, para la etapa I. En estadío II, $67 \%$ a 5 años y $55,8 \%$ a 10 años, estadío III $52 \%$ a 5 años y $34,7 \%$ a 10 años, estadío IV $50,6 \%$ a 5 años y $42,3 \%$ a 10 años.

La sobrevida a 5 años de tumores $\mathrm{T}_{1}$ fue de $87,5 \%, \mathrm{~T}_{2}$ de $69,4 \%, \mathrm{~T}_{3}$ de $34,54 \%$ y $\mathrm{T}_{4}$ de $53,26 \%$ (Figura 3); la sobrevida a 5 años de los pacientes según el estado de linfonodos cervicales fue: $\mathrm{N}_{0}$ $64,4 \%, \mathrm{~N}_{1}$ de $46,2 \%, \mathrm{~N}_{2}$ de $41,7 \%$ y $\mathrm{N}_{3}$ de $42,8 \%$. (Figura 4). 
SOBREVIDA GLOBAL Y POR ESTADÍOS DE 137 PACIENTES CON CÁNCER INTRAORAL. EXPERIENCIA DEL...

Tabla 1. Ubicación del tumor primario y diagnóstico histológico de 137 pacientes con cáncer intraoral

\begin{tabular}{lcclrr}
\hline Subsitio & & & & \\
Cavidad oral & $\mathbf{n}$ & $\mathbf{\%}$ & Tipos histológicos & n & \% \\
\hline Lengua & 55 & 40,1 & Carcinoma epidermoide & 116 & 84,7 \\
Piso de boca & 36 & 26,3 & Adenocarcinoma & 6 & 4,4 \\
Trígono retromolar & 13 & 9,5 & Carcinoma adenoideo quístico & 6 & 4,4 \\
Reborde alveolar & 11 & 8,03 & Melanoma & 2 & 2,2 \\
Paladar & 11 & 8,03 & Otras histologías & 7 & 4,5 \\
Mucosa mejilla & 11 & 8,03 & & 137 & 100 \\
Total & 137 & 100 & Total & & \\
\hline
\end{tabular}

n: Número de pacientes. \%: Porcentaje.

Tabla 2. Distribución de los 137 pacientes con cáncer intraoral según TNM y etapa de la enfermedad

\begin{tabular}{|c|c|c|c|c|c|c|c|c|c|c|c|}
\hline \multicolumn{3}{|c|}{ Tamaño tumoral } & \multicolumn{3}{|c|}{ Linfonodos cervicales } & \multicolumn{3}{|c|}{ Metástasis a distancia } & \multicolumn{3}{|c|}{ Etapa } \\
\hline (“T”) & $\mathbf{n}$ & $\%$ & (“N”) & $\mathbf{n}$ & $\%$ & (“M”) & $\mathbf{n}$ & $\%$ & & $\mathbf{n}$ & $\%$ \\
\hline $\mathrm{T}_{1}$ & 8 & 5,8 & $\mathrm{~N}_{0}$ & 83 & 60,6 & $\mathrm{M}_{0}$ & 135 & 98,5 & I & 7 & 5,1 \\
\hline $\mathrm{T}_{2}$ & 27 & 19,7 & $\mathrm{~N}_{1}^{0}$ & 16 & 11,7 & $\mathrm{M}_{1}^{0}$ & 2 & 1,5 & II & 22 & 16,1 \\
\hline $\mathrm{T}_{3}^{2}$ & 24 & 17,5 & $\mathrm{~N}_{2}$ & 24 & 17,5 & & & & III & 22 & 16,1 \\
\hline $\mathrm{T}_{4}^{3}$ & 78 & 56,9 & $\mathrm{~N}_{3}^{2}$ & 14 & 10,2 & & & & IV & 86 & 62,8 \\
\hline Total & 137 & 100 & Total & 137 & 100 & Total & 137 & 100 & Total & 137 & 100 \\
\hline
\end{tabular}

TNM se compone de (T): Tamaño tumoral, $(\mathrm{N})$ : Linfonodos regionales comprometidos, $(\mathrm{M})$ :

Tabla 3. Distribución de los 137 pacientes según tratamientos efectuados

\begin{tabular}{lcc}
\hline Tratamiento & $\mathbf{n}$ & $\mathbf{\%}$ \\
\hline Cirugía - RT & 85 & 62,04 \\
RT-QMT & 18 & 13,1 \\
Paliativo & 18 & 13,1 \\
Cirugía exclusiva & 4 & 2,9 \\
Cirugía rescate & 3 & 2,2 \\
RT exclusiva & 8 & 5,8 \\
Otras & 1 & 0,74 \\
Total & 137 & 100 \\
\hline n: Número de pacientes. \%: Porcentaje. RT: Ra- \\
dioterapia. QMT: Quimioterapia.
\end{tabular}



Figura 1. Sobrevida global en 137 pacientes con cáncer intraoral.

Tabla 4. Resumen de la sobrevida según estadíos en distintos estudios

\begin{tabular}{cccccc}
\hline Etapa & $\begin{array}{c}\text { Shaha et al. } \\
\mathbf{1 9 9 4} \mathbf{( \% )}\end{array}$ & $\begin{array}{c}\text { Hicks et al. } \\
\mathbf{1 9 9 7} \mathbf{( \% )}\end{array}$ & $\begin{array}{c}\text { Sessions et al. } \\
\mathbf{2 0 0 2} \mathbf{( \% )}\end{array}$ & $\begin{array}{c}\text { Woolgar et al. } \\
\mathbf{1 9 9 9} \mathbf{( \% )}\end{array}$ & $\begin{array}{c}\text { Sessions et al. } \\
\mathbf{2 0 0 0}(\mathbf{\%})\end{array}$ \\
\hline I & 88 & 95 & 76 & 85 & 72 \\
II & 80 & 86 & 67 & 90 & 63 \\
III & 66 & 82 & 39 & 82 & 44 \\
IV & 32 & 52 & 27 & 42 & 47 \\
\hline
\end{tabular}




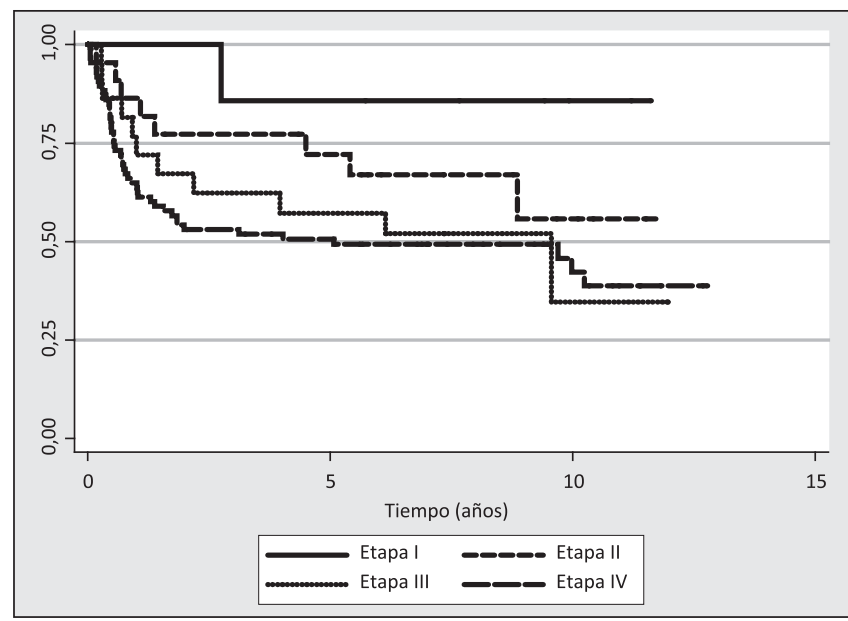

Figura 2. Sobrevida global en pacientes cáncer intraoral, por estadío.

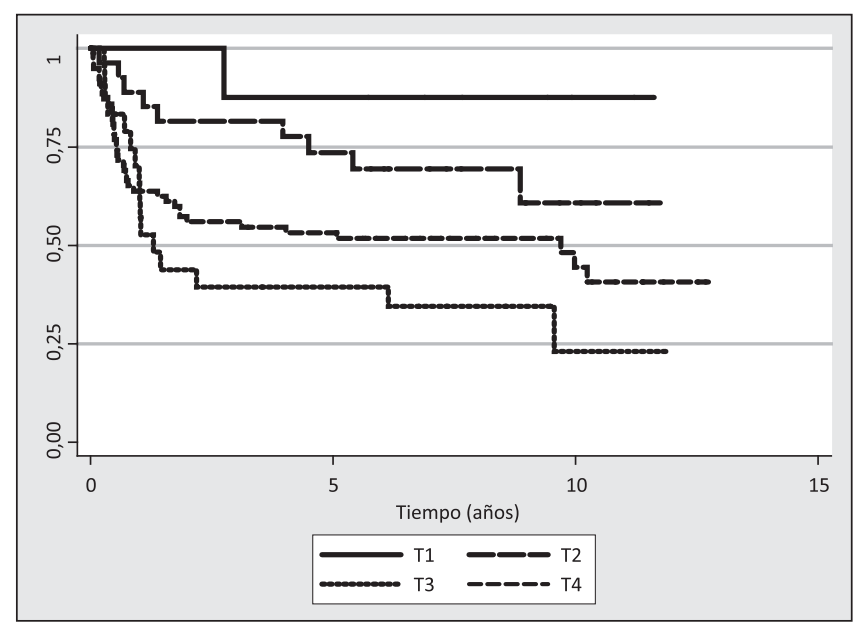

Figura 3. Sobrevida global en 137 pacientes con cáncer intraoral según tamaño tumoral.

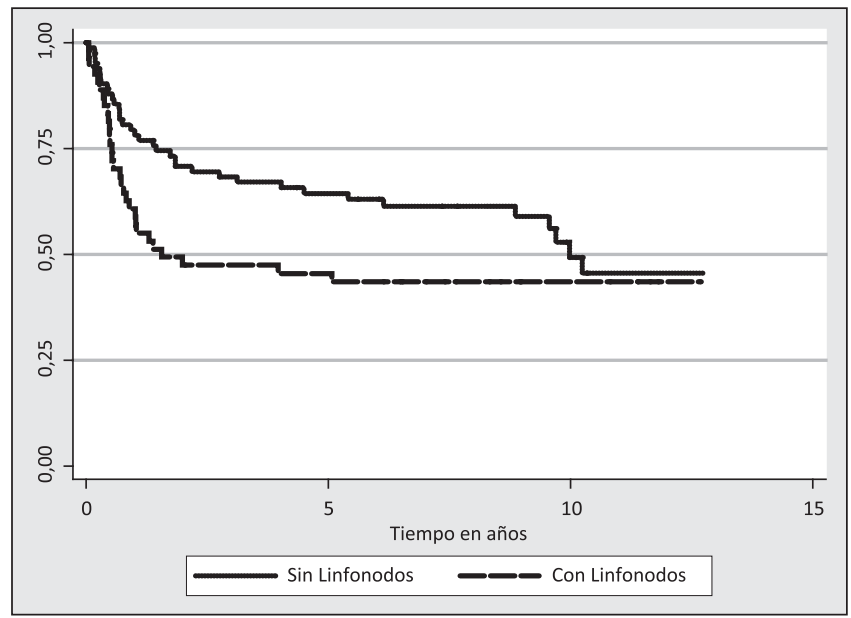

Figura 4. Sobrevida global en 137 pacientes con cáncer intraoral según estado de linfonodos cervicales.

\section{Discusión}

El INC es un centro terciario de derivación. Recibe pacientes derivados de la zona norte de Santiago y de las regiones IV a la VII, y con cierta frecuencia derivados de otras zonas del país. Así en este centro se atienden pacientes para realizarse el primer tratamiento o para tratar la recurrencia o la progresión de la enfermedad. De esta manera la población que compone esta serie es altamente heterogénea desde ese punto de vista. Sin embargo, esta es la realidad de otros centros similares de derivación, es por ello que el perfil demográfico, las características clínicas e histológicas de esta serie son comparables a otras series reportadas previamente ${ }^{1-3}$. Tal como se expuso anteriormente, la cirugía acompañada de radioterapia es el principal tratamiento realizado en este grupo de pacientes ${ }^{6}$.

Los resultados de la sobrevida global de nuestra serie pueden compararse con la de otros centros. En el estudio de Lung et $\mathrm{al}^{7}$, efectuado en una serie de 352 pacientes portadores de cáncer intraoral tratados entre 1993 y 2002 , la sobrevida global a 5 años fue de $31 \%{ }^{7}$. La explicación de esta baja sobrevida fue que la mayor parte de los pacientes tenían enfermedad avanzada y tumores con alto riesgo de recurrencia (cánceres indiferenciados y con compromiso linfático regional). En comparación a este reporte, nuestra sobrevida global a 1 año de seguimiento es peor en 10 puntos porcentuales, debido a que la recurrencia de nuestros pacientes es precoz. A los 3 años nuestra sobrevida es mejor $(59,3 \%$ vs $50 \%$ ) y se estabiliza desde los 5 años en adelante $(56,1 \%$ vs $46,3 \%)$.

Otro estudio ${ }^{8}$, comparó 2 poblaciones con cáncer intraoral de un país desarrollado (Memorial Sloan Kettering Cancer Center, New York) con otro en vías de desarrollo (AC Camargo, Sao Paulo) con poblaciones similares en edad y distribución del tumor primario. En comparación con esta serie, la sobrevida obtenida en nuestro estudio fue de $56,1 \%$ a 5 años, lo que se asemeja más a la obtenida en el país desarrollado $(61 \%)$ que a la del país en vías de desarrollo (43\%), sin embargo, la población de nuestro estudio tiene un menor número de pacientes con tabaquismo y un mayor número de pacientes mujeres que pudieran explicar esta diferencia. 
Carvalho et $\mathrm{al}^{9}$, estudiaron la sobrevida de 3.267 pacientes tratados durante 44 años, en él demuestra que la sobrevida en la década del 50 ' fue de $28,7 \%$, $29 \%$ en los 60 ', $26,4 \%$ en los $70^{\prime}, 32,5 \%$ en los 80 ' y $43,2 \%$ en la década de los 90 '. No obstante hay series con mejores resultados. Un estudio australiano logra una sobrevida global de 83,3\% en 116 pacientes con cáncer intraoral tratados entre 1991 y $2002^{10}$, similares en la distribución del tumor primario, sin embargo, esta serie tiene un mayor porcentaje de tumores precoces $(60 \%)$ vs tumores avanzados $(40 \%)$ y una baja cantidad de pacientes fumadores, que a la luz de estos resultados impactaría favorablemente en la sobrevida. Otras sobrevidas globales a 5 años en pacientes con cáncer intraoral son las descritas por Woolgard et $\mathrm{al}^{11}$, con un $64 \%$, Funk et $\mathrm{al}^{12}$, con un $49,8 \%$ y Chen et $\mathrm{al}^{13}$, con un $71 \%$.

En la Tabla 4 se resume la sobrevida por estadío de distintas series de pacientes con cáncer intraoral. La sobrevida en una serie distinta de 137 casos de cáncer intraoral avanzado $\left(\mathrm{T}_{4}\right)$ según estado de linfonodos cervicales fue de $34,5 \%\left(\mathrm{~N}_{\mathrm{o}}\right), 37,8 \%$ $\left(\mathrm{N}_{1}\right), 15,6 \%\left(\mathrm{~N}_{2}\right)$ y $0 \%\left(\mathrm{~N}_{3}\right)^{18}$, otra serie mostró una sobrevida de $57,9 \%, 19,6 \%, 21,1 \%$ y $1,4 \%$ respectivamente en tumores de piso de la boca, un subsitio de reconocido peor pronóstico ${ }^{17}$. En comparación a ellas, nuestra serie presentó mejores resultados debido probablemente a que incluyó pacientes con tumores precoces y avanzados de distintos subsitios de la cavidad oral. En ambas series la sobrevida se relaciona con el tamaño tumoral y con el estado de linfonodos cervicales.

Otras 2 series son comparables con la nuestra, la primera es la serie de Carvalho et $\mathrm{al}^{9}$, compuesta de 3.267 pacientes tratados durante 44 años, en ella la sobrevida de tumores precoces (estadíos I y II) y avanzados (estadíos III y IV) tratados con un abordaje multidisciplinario similar al de nuestra institución. Otra serie del mismo autor ${ }^{8}$, en que compara sus resultados con los de un centro de referencia en Nueva York (Memorial Sloan Kettering Cancer Center) también es comparable con los resultados aquí reportados.

\section{Referencias}

1. Riera P, Martínez B. Morbilidad y mortalidad por cáncer oral y faríngeo en Chile. Rev Med Chile 2005;133:55563.

2. Palma C, Gullane P, Gilbert R. Current treatment options in squamous cell carcinoma of the oral cavity. Surg Oncol Clin N Am. 2004; 13:47-70.

3. Tsantoulis PK, Kastrinakis NG, Tourvas AD, Laskaris
G, Gorgoulis VG. Advances in the biology of oral cancer. Oral Oncol. 2007;43:523-34.

4. Leite ICG, Koifman S. Survival analysis in a sample of oral cancer patients at a reference hospital in Río de Janeiro, Brazil. Oral Oncol. 1998;34:347-52.

5. Greene FL, Page D, Morrow M el al. AJCC Cancer Staging Manual, 6th ed. New York: Springer; 2002.

6. Capdeville F. Tumores malignos de la cavidad oral. Operación comando. Reconstrucción mandibular. Rev Chil Cir. 2005;57:7-18.

7. Lung T, Tăşcau OC, Almăşan H, Mureşan O. Head and neck cancer, treatment, evolution and post therapeutic survival-Part 2: A decade's result 1993-2002. J Cranio Maxillofac Surg. 2007;35:126-31.

8. Carvalho A, Singh B, Spiro R, Kowalski L, Shah J. Cancer of the oral cavity: A comparison between institutions in a developing and developed nation. Head Neck 2004;26:31-8.

9. Carvalho A, Ikeda M, Magrin J, Kowalski L. Trends of oral and oropharyngeal cancer survival over five decades in 3267 patients treated in a single institution. Oral Oncol. 2004;40:71-6.

10. Chandu A, Adams AC, Smith ACH. Factors affecting survival in patients with oral cancer: an Australian perspective. Int J Oral Maxillofac Surg. 2005;34:514-20.

11. Woolgar JA, Rogers SN, West CR, Errington RD, Brown JS, Vanghan ED. Survival and pattern of recurrence in 200 oral cancer patients treated by radical surgery and neck disecction. Oral Oncol. 1999;35:257-65.

12. Funk GF, Hynds-Karnell L, Robinson RA, Zhen WK, Trask DK, Hoffman HT. Presentation, treatment and outcome of oral cavity cancer: a national cancer database report. Head Neck 2002;24:165-80.

13. Chen YK, Huang HC, Lin LM, LinCC. Primary oral squamous cell carcinoma: an analysis of 703 cases in Southern Taiwan. Oral Oncol. 1999;35:173-9.

14. Shaha AR, Spiro RH, Shah JP, Strong EW. Squamous carcinoma of the floor of mouth. Am J Surg. 1994;148:455-9.

15. Hicks WL Jr, Loree TR, Garcia RI, Maamoun S, Marshall D, Orner JB, et al. Squamous cell carcinoma of the floor of mouth: a 20 year review. Head Neck 1997;19:400-5.

16. Sessions D, Spector G, Lenox J, Haughey B, Chao C, Marks J. Analysis of treatment results for oral tongue cancer. Laryngoscope 2002;112:616-25.

17. Sessions D, Spector G, Lenox J, Parriot S, Haughey B, Chao C, et al. Analysis of treatment results for floor-ofmouth cancer. Laryngoscope 2000;110:1764-72.

18. Tankéré F, Camproux A, Barry B, Guedon Ch, Depont J, Gehamco P. Prognostic value of lymph node involvement in oral cancers: A study of 137 cases. Laryngoscope 2000;110:2061-5. 